\title{
Linked color imaging and blue light imaging for evaluating a depressed-type adenoma in the colon
}

\author{
Shunsuke Yamamoto, Nima Mottacki \\ Sahlgrenska Academy, University of Gothenburg, Gothenburg, Sweden
}

A 70-year-old man with anemia attended the hospital for colonoscopy. The examination was performed using linked color imaging (LCI) and blue light imaging (BLI) with a highdefinition magnifying colonoscope (EC-760ZP-VM, Fujifilm Co.). White light imaging revealed a depressed-type polyp in the ascending colon, $8 \mathrm{~mm}$ in size, and was assessed as 0 -IIc according to the Paris classification (Fig. 1). LCI showed an enhanced image of the depressed morphology of the polyp (Fig. 1). Magnifying BLI revealed pits smaller than those seen in surrounding normal mucosa (type IIIs in Kudo classification). No signs of invasion, such as destructed pits or vessels with varied caliber, were seen (Fig. 1). Endoscopic mucosal resection was performed. The pathological diagnosis was tubular adenoma with low-grade dysplasia, as expected from the pretreatment observation, and it was completely resected (Fig. 2).

Flat- or depressed-type adenomas are rarely seen, accounting for less than 5\% of all adenomatous lesions in the colon. They harbor a significantly higher risk for invasive carcinoma than protruding-type adenomas [1]. For endoscopic evaluation of the polyps, dye solutions such as crystal violet or indigo carmine have traditionally been used. Whereas dye-

Department of Internal Medicine \& Clinical Nutrition, Institute of Medicine, Sahlgrenska Academy, University of Gothenburg, Gothenburg, Sweden

Conflict of Interest: S. Yamamoto has received an educational grant from Fujifilm unrelated to the content of this article

Correspondence to: Shunsuke Yamamoto, MD, PhD, Blå Stråket 3, S-413 45 Göteborg, Sweden, e-mail: shun0515suke@gmail.com

Received 17 February 2020; accepted 21 February 2020; published online 14 March 2020

DOI: https://doi.org/10.20524/aog.2020.0466

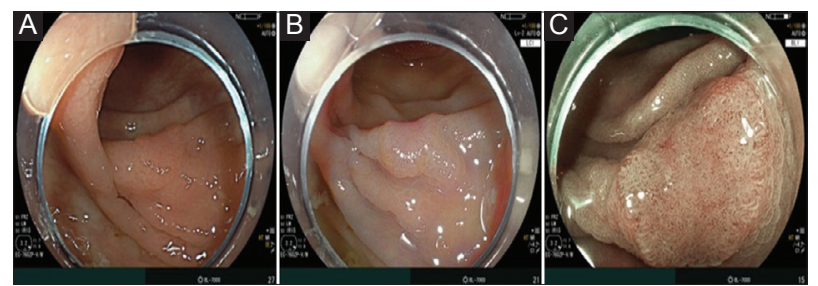

Figure 1 (A) White light imaging revealed a superficial depressed-type polyp. (B) Linked color imaging of the polyp without magnification. (C) Magnifying blue light imaging clearly visualized surficial pits in the lesion

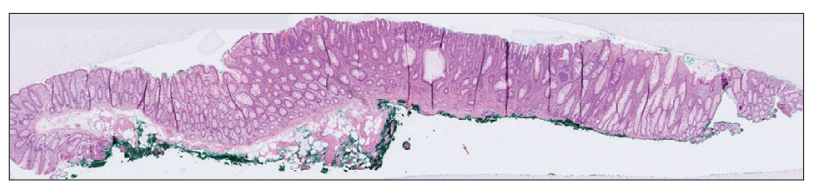

Figure 2 Histology of the resected polyp showing a tubular adenoma with low-grade dysplasia

based chromoendoscopy techniques may provide increased precision in predicting polyp histology, they require additional expertise and cost, and their application is time-consuming. The advent and development of high-resolution electronic chromoendoscopy might confine the use of these dye solutions to exceptional cases.

\section{Reference}

1. Yamamoto S. Depressed-type adenoma in the colon is associated with advanced pathologic changes. Gastrointest Endosc 2019;89:1262-1263. 